\title{
A narrative review of the impact of donor factors and selection criteria on outcomes after lung transplantation
}

\author{
Matthias Peltz \\ Department of Cardiovascular and Thoracic Surgery, University of Texas Southwestern Medical Center, Dallas, TX, USA \\ Correspondence to: Matthias Peltz. Department of Cardiovascular and Thoracic Surgery, University of Texas Southwestern Medical Center, 5939 \\ Harry Hines Blvd, POB 1, Suite HP10.110, Dallas, TX 75390-8879, USA. Email: Matthias.peltz@utsouthwestern.edu.
}

\begin{abstract}
Objective: The purpose of this review is to critically evaluate lung donor selection criteria and assess their impact on outcomes after lung transplantation.

Background: Lung transplantation remains the most effective therapy for end stage pulmonary disease. Selection of a suitable donor is essential for a successful recipient outcome. Both physiological and physical characteristics of the donor organ factor into decision making for proceeding with a transplant. Donor medical history may also be important when assessing potential offers.

Methods: A contemporary literature review of lung donor variables and their impact on outcomes after lung transplantation was performed using Ovid and PubMed search engines. The relative importance of each factor on decision making was evaluated based on the available evidence from identified reference sources.

Conclusions: Donor acceptance criteria typically involve acceptable gas exchange, size match, radiographic appearance, bronchoscopic inspection, limited smoking history and finally intraoperative surgical evaluation. Donor age, human leukocyte antigen (HLA) mismatches, serology and comorbidities such as hypertension and diabetes mellitus may be equally if not more important risk factors affecting recipient survival. Additionally, certain recipient variables should be considered when evaluating an organ offer. Donor type, brain dead or donation after determination of circulatory death, does not seem to significantly affect outcomes after lung transplantation.
\end{abstract}

Keywords: Lung donation; donor selection criteria; lung transplantation

Received: 01 February 2021; Accepted: 06 August 2021.

doi: $10.21037 /$ ccts-21-2

View this article at: https://dx.doi.org/10.21037/ccts-21-2

\section{Introduction}

Lung transplantation continues to be limited an inadequate donor supply. While the number of transplants performed has steadily increased, waiting list additions continue to outpace the number of available donor organs and waitlist mortality remains excessive. In 2018, an all time record of 2,562 transplants were performed in the United States. This number was easily eclipsed by the 3,134 patients added to the waiting list and the 362 candidates that died or were removed from waiting lists for being too ill. Waitlist mortality for patients with lung allocations scores (LAS) greater than 50 continues to exceed 100 per 100 patient years and mortality for patients removed from the waitlist for reasons other than being too ill is also not trivial (1).

During the early days of lung transplantation, donor selection criteria were stringent often restricting acceptable donors to ideal candidates (2). As experience with the procedure and management of recipients advanced and waiting lists increased, donor selection criteria have become less selective (3) and extended donors have been increasingly utilized $(4,5)$. Donor utilization was expanded from brain dead donors only to also include organs from donors recovered after circulatory determination of death (DCD). Donor acceptability criteria are largely based on expert opinion and have rarely been studied in a prospective manner. Most evidence relies on single center 
or registry data.

Changes in organ allocation that prioritize recipient acuity, such as the lung allocation score (LAS) in the United States, have been intended to provide the most critically ill with access to donor organs. Nonetheless, organ utilization for lungs continues to lag behind other organs with only around $20 \%$ of eligible organs transplanted, despite continued increases in the waiting lists (6). Some studies suggest that as many as $40 \%$ of rejected lungs might have been suitable for transplantation and argues for a more aggressive approach to evaluation of donor organs (7). Given the limited evidence and the number of discarded organs, there is the potential to recover additional donors. The advent of ex-vivo perfusion technologies has led to increased utilization of questionable organs or recovery of organs that were deemed unsuitable by conventional criteria (8).

Donor evaluation occurs in two distinct phases. First, upon receipt of an offer, an initial assessment of the organ is made based on available data. This includes circumstances surrounding the donor's cause of death and subsequent hospital course, comorbidities, past medical and surgical history, social history, laboratory data, relevant donor serology and importantly lung specific data including gas exchange, imaging and bronchoscopy $(9,10)$. The organ may be accepted or declined after initial review of the offer. More often, changes in donor management are requested or additional diagnostic data are obtained before arriving at a decision. Once a donor is accepted, the second phase occurs. This involves the intra-operative assessment of the donor during the organ recovery operation. The donor organ is evaluated to verify its suitability. This is not a trivial exercise and turndown rates for lungs due to unexpected intraoperative findings are higher than for all other major solid organs transplanted (6).

Standard donor and recipient factors that are used for determination of organ suitability are illustrated in Table 1. In reality, few donors actually meet these ideal criteria. Most transplant centers are willing to accept some abnormal findings when considering donor lungs. Additionally, many of the donor variables from donor selection guidelines do not seem to affect recipient survival after transplantation (11). In this review donor factors and their impact on recipient outcomes will be explored. We present the following article in accordance with the Narrative Review reporting checklist (available at https:// ccts.amegroups.com/article/view/10.21037/ccts-21-2/rc).

\section{Methods}

A contemporary English language literature review of lung donor variables and their impact on outcomes after lung transplantation was performed using Ovid and PubMed search engines from the time period of 1980 to June 2021. Initially searches were performed using the terms "lung donor" and "lung transplantation". Queries were further refined to allow for focused assessment of identified donor variables. The relative importance of each factor on decision making was evaluated based on the available evidence from identified reference sources.

\section{Discussion/summary}

\section{Narrative - donor factors}

\section{Gas exchange}

Oxygenation or gas exchange are often considered the most important physiological parameter when assessing donor lung function. An arterial partial pressure of oxygen $\left(\mathrm{PaO}_{2}\right)$ to fractional inspired oxygen $\left(\mathrm{FiO}_{2}\right)$ ratio of $>300$ is typically considered the minimally acceptable ratio. Thabut et al. in 2005 in a multi-center study from France reported that recipient outcomes were worse when the donor $\mathrm{PaO}_{2}: \mathrm{FiO}_{2}$ ratio was <350 (12) even when adjusting for several other risk factors. However, donor gas exchange is influenced by a number of variables and it should not be viewed in isolation and at a single time-point when considering suitability of a donor. Ventilator parameters can influence gas exchange. These should be reviewed and recruiting maneuvers performed if gas exchange is not acceptable. Recruitment protocols have been shown to improve donor gas exchange and increase recovery of donor lungs. In a study by Chang et al. donor lung recovery after implementation of a lung protective recovery protocol improved from $19.8 \%$ to $33.9 \%$ despite lower initial $\mathrm{PaO}_{2}$ and more donors with an initial $\mathrm{PaO}_{2}<200 \mathrm{mmHg}$ (13). In one of the only randomized studies on lung donor recovery, Mascia et al. demonstrated that a protective ventilator protocol improved donor eligibility by $41 \%$ and increased the number of organs transplanted by $27 \%$ compared to conventional management with a significant increase in $\mathrm{PaO}_{2}$ in the protective lung ventilation group (14).

Other studies, including the International Society for Heart and Lung Transplantation (ISHLT) 2020 donor specific annual report did not note a difference over a range of $\mathrm{PaO}_{2}: \mathrm{FiO}_{2}$ ratios. While the denominator of 
Table 1 Standard lung donor acceptance criteria

\begin{tabular}{|c|c|}
\hline Criteria & Findings \\
\hline ABO group & Identical or compatible \\
\hline Gas exchange & $\mathrm{PaO}_{2}: \mathrm{FiO}_{2}>300$ \\
\hline Imaging (chest X-ray, CT scan) & Clear lung fields \\
\hline \multirow{3}{*}{ Bronchoscopy } & No or minimal airway erythema or edema \\
\hline & Normal bronchial anatomy \\
\hline & No aspiration \\
\hline Microbiology & No organisms on Gram stain or culture \\
\hline Medical history & No chest trauma \\
\hline Surgical history & No prior cardiopulmonary surgery \\
\hline Size & Appropriate size match for recipient based on donor height/weight and available imaging \\
\hline \multirow[t]{5}{*}{ Intraoperative evaluation } & Normal lung recoil \\
\hline & No emphysema \\
\hline & No evidence of lung injury or significant contusion \\
\hline & No lung nodules \\
\hline & No or minimal edema \\
\hline
\end{tabular}

total donors evaluated is often not known, in the ISHLT registry report, 6,769 out of $24,123(28.1 \%)$ of all donors recovered had a $\mathrm{PaO}_{2}: \mathrm{FiO}_{2}$ ratio <300. Both 1 year and 5 -year conditional survival of these donors was not different from donors with a $\mathrm{PaO}_{2}: \mathrm{FiO}_{2}$ ratio $>300$ suggesting a significant number of donors with relatively reduced $\mathrm{PaO}_{2}$ are suitable for transplantation (15). Difficult to recruit atelectasis by conventional ventilator maneuvers has led some centers in a recent study to assess donor lungs after performing intraoperative recruitment maneuvers. While applied to donors with body mass indices over 25 , this strategy seems applicable to most donors in the absence of other contraindications to transplantation (16). Also, when unilateral lung disease potentially affects gas exchange, this does not necessarily exclude the contralateral lung as a suitable organ. The advent of ex-vivo lung perfusion
(EVLP) suggests many donors continue to be declined for impaired oxygenation and the use of ex vivo perfusion technology is useful for determining acceptability of these organs (8). A recent prospective multi-center study using a standardized intraoperative ventilator protocol suggested that intraoperative gas exchange and $\mathrm{PaO}_{2}: \mathrm{FiO}_{2}$ ratio of accepted lungs did not correlate with short term outcomes after transplantation (17).

\section{Age}

The suggested ideal lung donor is less than 50 to 55 years of age (18). A minimum donor age is not typically considered a contraindication and size considerations are more important factors when assessing teenage or pediatric donors though at least one study suggests donors under the age 18 years may be associated with increased 1 year mortality after 
transplantation (19). The effect of increasing donor age on outcomes after transplantation has received considerable attention. Both increased early and late mortality were reported in early studies evaluating the impact of donor age (7). The mortality risk seems to be particularly increased in older and more critically ill recipients $(19,20)$. Older data suggested that there appeared to be an interaction of longer donor ischemic time ( $>7$ hours) and mortality in older donors (> age 50) (21). In contrast, a recent analysis by Mulvihill et al. of United Network for Organ Sharing (UNOS) data, demonstrated no difference in survival by ischemic time in older donors even when the ischemic time exceeded 8 hours (22). The International Society for Heart Lung Transplantation focus issue on donor characteristics from 2020 divided donor age into terciles (18-34 years, $35-49$ years, 50 years or older). In this largest registry study to date, donor age was associated with increased 1 year mortality and while different for all recipient age terciles (18-39 years, $40-59$ years, 60 years or older), this only began reaching statistical significance for recipients 40 years of age or older. There was no effect on 1 year conditional survival across all donor and recipient age groups (15). Others have reported that most of the increased mortality risk is mainly associated with donors older than $60-65$ years. Multivariable adjusted hazard ratios ranged from 1.44 for donors aged 60 or older (20) to 2.33 for donors age 65 or older when restricted to the LAS era (23). This effect seemed to be associated with both reduced short and long-term survival (22). The overall impact of donor age must be balanced against the potential recipients expected wait list mortality and likelihood of another offer when evaluating older donors.

Many potentially suitable lungs from older donors are rejected. Age is the most common reason for not even obtaining consent for donation among eligible deaths over the age of 55 (24). Comparing donor conversion rates from over 160,000 donors in the United States, lungs from 22.8\% of doors $<55$ years of age were transplanted compared to only $8.9 \%$ for donors over 55 years of age. Lungs from DCD donors were particularly underutilized even though based on registry and single center studies (24), there has not been any compelling evidence that donor type impacts outcomes in older donors (25). In the 2019, ISHLT Registry Report, while there is an association between donor age and recipient mortality, this effect appears small and linear up to a donor age of 60 , compared to the more exponential increase in mortality beginning at a recipient age of 50, particularly when 5 - and 10-year survival are considered (26).

\section{Microbiology}

Post-transplantation pneumonia is associated with increased recipient mortality (27). Donor derived infections are felt to contribute to its development. Sputum cultures and Gram-stain are routinely obtained from potential lung donors in the United States. Positive cultures and stains are not uncommon though implications of these findings on recipient outcomes are not entirely clear. Many centers also routinely obtain donor and recipient airway cultures at the time of the implant to guide antibiotic therapy yet these do not seem to correlate with development of post-transplantation pneumonia (27-29). An early report suggested that positive donor broncho-alveolar lavage (BAL) was associated with worse recipient outcomes though the mechanisms were not entirely clear since only one out of 53 recipients ( $46 \%$ of the cohort) with positive BALs developed a donor derived thoracic infection (28). Bonde et al., on the other hand, found no such association (27). In another more recent large single center study, the incidence of a positive donor respiratory culture was over $50 \%$ but donor derived pulmonary infection, mainly tracheobronchitis, occurred in only $2.9 \%$ of recipients and was managed with antibiotic therapy tailored to the isolated organism (29).

\section{DCD}

Potential lung donors may be of three types: (I) brain dead; (II) DCD; (III) living donor. For purposes of this review, will only consider deceased donation. Although most donors continue to be from brain death donors, transplantation of lungs recovered from DCD donors has increased recently. The vast majority of DCD donors are recovered under controlled circumstances (Modified Maastricht Categories 3-5). Fewer than one percent are transplanted from donor organs recovered from category 1 and 2 uncontrolled DCD donors (25). Recovery rates from DCD donors lag behind brain dead donors. Aside from a general assessment of donor quality, other factors are important when considering acceptance of DCD donors. These include use include time from withdrawal of life sustaining therapy (WLST) to cardiac arrest and time to pulmonary flush. Use of pre-mortem heparin, definition of circulatory death, stand-off period, timing of bronchoscopy and management of the endotracheal tube may vary widely among hospitals for recovery of controlled DCD donors. The acceptable time from WSLT to cardiac arrest is not fully established. It is to some extend influenced by donor saturations and the period after cessation of effective perfusion (systolic blood pressure $<50 \mathrm{mmHg}$ ) but generally ranges from 60 up to 
90 minutes $(25,30)$. Initiation of cold flush should occur within 30 minutes of ineffective perfusion but this interval by definition is often prolonged in the uncontrolled DCD setting. In a recent multi-center review, warm ischemic times exceeding 60 minutes as measured by cessation of effective perfusion, did not result in worse 30 -day and 1 year survival (31). Some centers have restricted utilization of DCD donors to local recoveries due to concerns about the impact of ischemic time on outcomes from these donors (32). Sabashnikov et al. reported that while longterm survival was similar, early graft function was worse and the risk of developing bronchiolitis obliterans syndrome (BOS) was higher in a propensity-matched study from their institution (33). On the other hand, experience by others does not indicate an increased risk of chronic allograft dysfunction in DCD donors (34). Interestingly, both these centers contributed to the ISHLT DCD Registry Report from 2019 that reported virtually identical survival between DCD and brain dead donors with no association of donor ischemic time or donor cause of death in either group (25). A recent report from Australia suggests that logistics and resource requirements may be more important reasons for underutilization of distant DCD donors compared to brain dead donors than concerns regarding ischemic time or postoperative outcomes (35).

Current data suggests outcomes from DCD donors are not substantially different from brain dead donors. Difficulty in fully assessing lungs from DCD donors may be one reason for reduced recovery rates. EVLP offers the opportunity to more fully evaluate the donor organ in this setting before making a final acceptance decision. In the ISHLT DCD registry report, nearly $20 \%$ of all donors were transplanted after EVLP (25).

\section{Cause of death}

Donor causes of death can be broadly categorized into traumatic brain injury, stroke/intracranial hemorrhage, anoxia, cardiovascular, and rarely others, such as central nervous system tumors. A number of studies indicate that donor mechanism of neurologic injury does not impact outcomes after lung transplantation $(25,36)$. Other reports have suggested some subtle outcome differences based on the donor cause of death. Both the 2019 and 2020 ISHLT Annual Reports noted a slight reduction in 1 year conditional survival for donors with traumatic brain injury compared the stroke as a cause of death $(15,26)$. While Ciccone et al. did not note a survival difference, rejection and BOS free survival in recipients of donor organs that suffered a traumatic brain injury was reduced in their single center report (37). Alternatively, a recent review of donor cause of death from the Organ Procurement and Transplantation Network (OPTN) suggested that mortality in donors dying from stroke was increased by univariate analysis and decreased in donors dying from anoxia by multivariable analysis compared to traumatic brain injury donors (38). Reyes et al., using UNOS data to develop a new risk model, suggested that traumatic brain injury in their model was protective (11). Overall, it is difficult to draw conclusions if donor mechanism of injury has a consistent effect on recipient outcomes. However, even if present, this effect is likely small and lacks relevance for clinical decision making.

Causes of anoxia are diverse and may include drug overdose, hanging, drowning, status asthmaticus, among others. Death due to asphyxia from underlying lung disease or airway obstruction has often been considered a contraindication to transplantation. While outcomes from donors with severe asthma have been poor (39), a number of more recent reports suggest that donors with other causes of acute airway obstruction can be transplanted. Death from hanging and other causes of acute airway obstruction are thought to cause post-obstructive barotrauma and negative pressure pulmonary edema that historically made these donors poor candidates for transplantation (40). Several studies, however, demonstrated that in otherwise suitable donors, outcomes from these donors are no different than from conventional donors $(40,41)$. In both studies, these donors represented nearly $10 \%$ of all transplants performed at these centers, suggesting wider utilization these donors could have a significant impact on transplant volumes. Death by drowning occasionally can also lead to good transplant outcomes as suggested by a UNOS/OPTN review from 2014 by Whitson et al. Hanging (1.5\%) and drowning $(0.2 \%)$ donor causes of death represented fewer than $2 \%$ of all transplants performed in this study, implying that these donors continue to be underutilized (36).

\section{Medical and surgical history}

Donors with underlying lung disease are not usually considered for transplantation except in select circumstances. While transplants performed from donors that died from asthma related complications seem to do poorly, donors that died from other causes and have a history of medically controlled asthma may be more suitable candidates. Oto et al., demonstrated that medically treated or not on treatment asthmatic donors had similar 
short and long-term survival to donors without a history of asthma (39).

Non-pulmonary donor comorbidities are often overlooked when evaluating potential donors. However, registry data suggest some may be important variables affecting recipient outcomes. Donor diabetes has been associated with worse recipient outcomes in multivariable analysis in a number of studies $(26,42)$. The magnitude of this effect appears equal to or greater than the reduced survival for recipients of lungs from older donors or donors with a significant smoking history $(11,15)$. The association of hypertension in the donor with increased recipient mortality is less strong but seems consistent, as well $(15,26)$. Long-term left heart disease can lead to pulmonary hypertension in the donor. Donor echocardiogram may suggest indirect signs of pulmonary hypertension such as right ventricular dysfunction or dilation and elevation of estimated right ventricular systolic pressure that would warrant further investigation. Underlying kidney disease, particularly end-stage renal disease may have important impact on lung function. While there are reports of successful transplants from donors with end-stage renal disease (43), clinical studies suggest that impairment of lung function is not uncommon in chronic kidney disease and may not be readily apparent when evaluating a potential donor even when gas exchange seems acceptable (44). A donor history of prior major cardiac or thoracic surgery is often considered a relative contraindication to lung recovery. However, some reports suggest that carefully selected, these donors can be recovered with good outcomes (45). A chest CT is recommended to assess the reentry and lung injury risk before recovering these donors.

\section{ABO compatibility and HLA matching}

Adult lung transplants are performed with $\mathrm{ABO}$ blood type identical or compatible donors. In the United States, donor lungs are allocated preferentially to $\mathrm{ABO}$ identical donors independent of recipient severity of illness as measured by the lung allocation score. The reasoning remains obscure. The ISHLT 2003 review of lung donor acceptability criteria and 2019 annual reports suggest a minor survival advantage in recipients of an ABO identical allograft $(18,26)$ though this finding has not been supported by other studies (23). Regardless, ABO compatibility versus $\mathrm{ABO}$ identical transplant is almost never used as a reason to decline an otherwise suitable donor and, this at best small benefit is dwarfed by the increasing wait list mortality among critical ill candidates.
Human leukocyte antigen (HLA) matching between donor and recipients for lungs is impractical based on the available number of donors. Centers generally do not consider the degree of HLA compatibility when assessing donors and typically ensure only exclusion of unacceptable donor specific antibodies. However, even single HLA locus matches may offer a survival benefit. Brugière et al. reviewed their institutional experience and noted that 0 or 1 HLA-A mismatches were associated with both improved short and long-term survival and resulted in a reduction in BOS compared to mismatch for both HLA-A haplotypes [hazard ratios (HR) of 1.97 for survival and 1.75 for bronchiolitis obliterans] (46). ISHLT Registry reports have routinely demonstrated a long-term survival advantage for recipients of better HLA matched donor organs (Chambers, Chambers). Our analysis of 9,791 transplants in the Organ Procurement and Transplantation Network data base demonstrated improved survival, reduced incidence of acute rejection, and development of bronchiolitis obliterans syndrome depending on the donor-recipient HLA match. In this study, the total HLA (4 or greater) and Class I mismatches correlated with worse survival and increased development of BOS at 5 years posttransplantation compared to allografts with 3 or fewer HLA mismatches. Also, zero DR mismatches between the donor and recipient seemed to be associated with a reduced incidence of reported acute rejection. The adjusted relative risk reduction in mortality ranged from hazard ratios of 0.62 ( $0-1$ mismatches) to 0.80 ( 3 mismatches) compared to a 6 mismatch graft in this review and the absolute survival advantage of $0-1$ mismatch allografts exceeded $15 \%$ compared to recipients of an allograft with 4 or more mismatches. These data suggest that the longterm survival benefits of even partially matched allografts are not inconsequential (42). Unlike some other donor variables discussed in this review, HLA matching appeared to mostly affect intermediate and long-term outcomes after transplantation.

\section{Donor-recipient size matching}

Donor height range is typically used for candidate listing and serves as a surrogate marker for acceptable donor lung size for any given recipient. This obviously is an imperfect measure since lungs occupy a volume within the chest which is truly the parameter of interest when considering acceptable organs. Nonetheless, donor height has implications for outcomes after transplantation. These to some extent are influenced by recipient diagnosis. Not 
surprisingly, donor height tends to be smaller for restrictive lung disease compared to obstructive lung disease. This topic was the recent focus of the 2019 Annual ISHLT report. Chronic obstructive pulmonary disease (COPD), $\alpha-1$ antitrypsin deficiency and cystic fibrosis candidate diagnosis donor height difference ranged from plus $4.1-6.4 \mathrm{~cm}$ compared to the recipient height for single and double lung transplants whereas donors recovered for recipients with interstitial lung disease patients tended to be slightly undersized by $0.5-1.9 \mathrm{~cm}$. Donor and recipient heights were similar for other diagnoses. Oversizing by donor height appeared to improve recipient 5 -year survival in the entire cohort but this benefit was only apparent in patients with COPD/alpha-1 antitrypsin deficiency and cystic fibrosis. There was a non-significant trend towards worse survival when donor height exceeded recipient height by more than $15 \mathrm{~cm}$ in interstitial lung disease patients. This report also suggested that lower donor weight compared to the recipient may be associated with worse survival though this was not explored further by recipient diagnosis (26).

Donor height and weight ranges are poor surrogates for lung volume and while useful for candidate listing, probably should not be used for decision making, at least in isolation. All donors have chest X-rays (CXR) available for review and increasingly, also chest computed tomography (CT) scans. While CXR provides a reasonable two-dimensional comparison of donor and recipient lung sizes, it does not account for anterior-posterior depth or the volume occupied by mediastinal structures. CT scan addresses these limitations. While thoracic volume can be accurately calculated from reconstructed CT imaging (47), this is not usually feasible real time for donor evaluation. We have utilized a frustrum model based on CT data for candidates with interstitial lung disease to estimate donor lung volume and compared this to recipient data (48). Donor ventilator settings and modes should be kept in mind particularly if there are discrepancies in sizing by different imaging modalities.

Many centers compare either actual or predicted total lung capacity (aTLC or pTLC, respectively) of the recipient to the pTLC of the donor using various formulae. The ISHLT 2003 guidance document suggests donors with a predicted TLC in the range of $75-125 \%$ should be considered acceptable size matches for most recipients with oversizing favored for recipients with obstructive diseases and undersizing preferred for recipients with restrictive lung disease (18). In an analysis of the Scientific Registry of Transplant Recipients (SRTR) in the LAS era, increasing donor to recipient pTLC ratio was associated with a decreasing risk of one year mortality from a ratio of 0.5 to 1.3 with a higher risk as the pTLC ratio increased further (49). This effect appeared independent of diagnosis. Eberlein $e t a l$. also suggested that any gender outcome differences were no longer significant when adjusted for donor to recipient pTLC ratio.

Others have reported that the use of recipient aTLC or pTLC may be preferable depending on the etiology of the underlying lung disease. Mason et al. reviewed their center's experience comparing these to the pTLC of the donor. While there was no difference in survival in their entire cohort over a wide range of donor-recipient ratios by either $\mathrm{pTLC}_{\text {donor }} / \mathrm{pTLC}_{\text {recipient }}$ (range, 0.55-1.59) or $\mathrm{pTLC}_{\text {donor }} / \mathrm{aTLC}_{\text {recipient }}$ (range, 0.52-4.20), survival was worse in emphysema patients receiving oversized or severely undersized organs based on $\mathrm{pTLC}_{\mathrm{donor}} / \mathrm{aTLC}_{\text {recipient }}$ $(>1.03$ or $<0.67)(50)$. Our experience using the Frustrum model suggested improved survival in recipients with interstitial lung disease of lungs with $\mathrm{pTLC} \mathrm{C}_{\mathrm{donor}} /{\mathrm{p} T L \mathrm{C}_{\text {recipient }}}$ over 1.0 though the need for diaphragm plication was increased in this population (48). A meta-analysis on donor - recipient size matching by Barnard et al. offered some generalized recommendations on this topic including (I) Multidisciplinary selection committee review of imaging studies and pulmonary function tests to determine acceptable donor pTLC range. (II) Use of recipient aTLC for COPD/emphysema candidates and not oversizing or severely undersizing organs. (III) Avoidance of aTLC for interstitial lung disease patients with guidance for acceptable donor pTLC based on review of pulmonary function testing and radiologic studies. (IV) Use of $\mathrm{p} T L \mathrm{C}_{\text {donor }} / \mathrm{pTLC}_{\text {recipient }}$ for diagnoses not expected to affect pTLC (51).

Airway sizing in adults is not often an issue. The diameter of the main bronchi from teenage or pediatric donors can be substantially smaller than adult recipient airways and requires further investigation to ensure an acceptable size match. Airway sizes may be estimated from chest X-ray but CT scan should be used for more accurate measurements. Our center performs most bronchial anastomoses by telescoping the smaller airway into the larger bronchus which can accommodate substantial size differences between donor and recipient airways. Airway complications in our experience with this technique are low though some other centers have reported increased adverse event rates with telescoped anastomoses (52). Other techniques, such as the "upper lobectomy" method, have been reported for managing extreme airway size mismatches though surgeon 
experience needs to considered when evaluating offers with severe donor-recipient airway discrepancies (53).

\section{Smoking/substance abuse}

Although the incidence of smoking continues to decrease in most countries, use of other controlled substances is increasing (15). According to recommended guidelines, standard criteria donors should have less than a 20 pack year smoking history $(7,11,18)$, but a more extensive history does not necessarily preclude utilization of the organs for transplantation. A number of studies have evaluated the impact of donor smoking history on outcomes after lung transplantation. While in general outcomes are inferior to transplants from nonsmokers (15), this difference is along the lines of other factors considered in this review and should not preclude recovery of these organs if other donor parameters are otherwise acceptable, particularly when wait list mortality is expected to be increased. Bonser et al explored this issue in a review of the United Kingdom Registry. The use of a donor with a smoking history was associated with an increased peri-operative, 1-, 3- and 5-year mortality compared to recipients of a nonsmoker allografts. The adjusted hazard ratio (HR) for mortality in this study was 1.36 at 3 years. However, modeling also suggested that wait list mortality was significantly reduced (HR 0.79) by accepting a donor with a smoking history instead of waiting for organs from a donor without a smoking history (54). In a single center review by Okahara and colleagues, active smoking seemed to be associated with reduced 3-year survival (HR 2.13) (55). In both these studies, the smoking history of donors was relatively modest which raises the question whether any donor with a smoking history can be considered "standard".

Donor use of alcohol and other substances such as opiates, cocaine, amphetamines, and marijuana does not seem to lead to clinically important differences in survival compared to conventional donors $(15,56)$. However, for donors with a significant history of cocaine or amphetamine use, donor EKG and echocardiogram should be reviewed carefully for any signs of pulmonary hypertension (56). While donor marijuana use in itself does not seem to affect outcomes after transplantation, at least one recent study suggests that marijuana inhalation in conjunction with smoking, may have an additive adverse affect on recipient survival (HR 2.97) and use of donors that smoke tobacco products and marijuana needs to be considered carefully (55).

\section{Hepatitis C infection}

Until recently, transplantation of lungs from hepatitis C seropositive donors was rarely performed and associated with worse recipient outcomes compared to transplants performed from uninfected donors. The development of direct acting anti-retrovirals for treatment of hepatitis $\mathrm{C}$ infection with published cure rates exceeding $95 \%$ has led to renewed interest in recovery of organs from these donors. Reported hepatitis $\mathrm{C}$ prevalence in donors ranges from $3.9 \%$ in Spain to $11.8 \%$ in Taiwan and thus represents an underutilized donor pool. Transmission from antibody positive but nucleic acid test negative donors is felt to be negligible whereas recipient infection is near $100 \%$ for organs received from nucleic acid testing positive donors. Both prophylactic and preemptive treatment regimen are affective in achieving a sustained viral response (57). Single institution studies in the current era are encouraging and suggest short-term recipient outcomes form hepatitis C viremic donors compare favorably to transplants from standard criteria donors (58). Longer term follow-up is required before fully advocating for use of these donors to ensure that these results are maintained since survival differences form untreated hepatitis $\mathrm{C}$ infected recipients did not emerge until three years after transplantation (57).

\section{Re-transplantation}

Re-transplantation has represented $2.6-8.3 \%$ of all lung transplants since 1990 according to the 2019 ISHLT registry report (26). Outcomes are inferior to primary transplants largely related to an increased early mortality (59). Considering the complexities of these reoperations, intuitively one would assume that centers would be more selective choosing donors. In practice, however, both from registry and single center data, no significant differences in donor variables are generally noted comparing primary lung transplants to retransplantation. While outcomes in retransplant recipients over 60 seem worse, no clear consensus about donor selection criteria has been established (58). Single institution reports do recommend that common donor specific antigens and undersizing of donors should be avoided $(60,61)$.

\section{Intraoperative evaluation}

While the previous donor factors influence whether acceptance of any given lung is being considered, ultimately the decision to move forward with a transplant relies on the 
intraoperative evaluation and judgment of the recovering surgeon. Nearly $20 \%$ of all donor lungs are declined in the operating room due to adverse findings even though preoperative evaluation was favorable (6). A detailed description of the donor recovery process is beyond this review.

In brief, the donor diagnostic evaluation and history are again reviewed along with up to date clinical data particularly the most recent arterial blood gas and chest $\mathrm{X}$-ray. Bronchoscopic evaluation is then performed evaluating for purulent secretions, repooling of secretions, airway inflammation and cobble stoning, blood, evidence of aspiration, airway trauma and anatomic abnormalities $(9,10)$. The majority of donors have some abnormalities on bronchoscopic evaluation and only about $20 \%$ of potential donors have normal gas exchange, clear chest $\mathrm{X}$-ray, and normal bronchoscopy (62). The impact of bronchoscopic and radiographic findings on recipient outcomes have not been studied in a prospective manner. These are best used as part of an overall assessment of organ quality and not a sole reason for declining a donor organ (63).

Sternotomy is then performed and the donor lungs are inspected. Donors with extensive pleural adhesions are frequently declined due to risk of prolonged air leaks associated with extensive adhesiolysis. Each lung is individually inspected and palpated for evidence of pneumonia, consolidation, edema, atelectasis, pulmonary emboli, nodules, emphysema or trauma. The donor is disconnected from the ventilator and lung collapse as well as recoil are assessed as surrogate markers of lung compliance. This assessment relies largely on surgeon experience and by its nature, is somewhat subjective. Wedge resections of peripheral nodules and other abnormalities can be performed if pathology support is available at the donor facility. The use of ultrasound to objectively quantify extracellular lung water content and consolidation has been reported for evaluation of the immediately recovered lungs and lungs evaluated with EVLP (64). Intraoperative pulmonary vein gas measurements have been investigated and seem to be more closely associated with bronchoscopic findings, parenchymal abnormalities and lung compliance during in vivo donor lung evaluation than preoperative studies (65). Additionally, protocol driven, intraoperative objective measures of lung elasticity, such as peak airway pressures and dynamic lung compliance described by Benazzo et al. may be more important predictors of early lung function than intra-operative gas exchange (17).

\section{Conclusions}

A comprehensive understanding of the impact of donor related factors on recipient survival is essential to make an informed decision regarding the suitability of a potential donor for a particular recipient. Among the standard lung donor selection criteria, only donor age and smoking status seem to adversely affect recipient survival. Other less commonly considered donor factors may be more important determinants of outcomes after transplantation. See Table 2.

While the focus of this review is on the impact of donor risk factors on recipient survival, especially for critically ill recipients, recipient factors are often as or even more important determinants of outcomes (66). Many centers are willing to accept a marginal organ for these candidates though this practice may adversely affect outcomes. In practice, the LAS score prioritizes these patients and in some circumstances, it may be advantageous to await a lower risk donor to not compound recipient risk. For example, a better size matched graft or younger donor seem to offer improved survival for higher LAS score candidates $(19,49)$. Scoring systems have been developed to guide acceptance of donor lungs. The recovery of donor lungs seems improved when an objective scoring system incorporating many of the parameters in this review are used in the donor evaluation process (67).

Many organs are declined without ever evaluating the donor organ in vivo. Data does suggest that some of these organs are useable. The advent of ex vivo perfusion technologies mitigates some of the risk of extended or marginal donors by allowing for a more thorough evaluation of the organ prior to implantation. A more aggressive approach to donor evaluation must be balanced against the resources required especially if organs are not local. The increasing use of dedicated organ recovery facilities by organ procurement organizations may avoid costs associated with long-distance travel to assess a marginal organ. Some organ procurement organizations have partnered with ex vivo technology manufacturers to make EVLP available to accepting centers when organ quality is in question. Alternatively, the use of organ recovery facilities, such as United Therapeutics, Inc. (Silver Spring, MD, USA) are another model to assess donor lung function after procurement before making a final decision regarding acceptance. How EVLP fits into the allocation process and cost sharing for organs that are declined on EVLP remain challenges that need to be addressed. 
Table 2 Impact of donor factors on recipient survival

\begin{tabular}{|c|c|}
\hline Donor criteria & Effect on outcomes/recommendations \\
\hline Gas exchange & In selected patients, no clear association with recipient outcomes \\
\hline Imaging & No effect on outcomes in isolation \\
\hline Donor type - DCD versus DBD & No difference in outcomes by donor age or ischemic time \\
\hline Bronchoscopy & No effect on outcomes in isolation \\
\hline Microbiology & No effect on outcomes in isolation; poor predictor of post-transplant pneumonia \\
\hline Prior cardiopulmonary surgery & Potentially recoverable in select donors \\
\hline \multirow[t]{3}{*}{ Size matching } & $\begin{array}{l}\text { COPD/Emphysema/ } \alpha 1 \text { ATD - avoid any oversizing or severe undersizing (donor pTLC to } \\
\text { recipient aTLC ratio }>1.0 \text { or }<0.67 \text { ) }\end{array}$ \\
\hline & Donor pTLC +/- 15-20\% of recipient (aTLC+pTLC)/2 \\
\hline & Other diagnoses - donor pTLC +/- 15-20\% of recipient pTLC \\
\hline ABO identical versus compatible & Likely no clinically significant difference \\
\hline HLA mismatches & Improved long-term survival for $0-3$ versus a greater number of HLA mismatches \\
\hline Diabetes mellitus & Increased recipient mortality risk \\
\hline Hypertension & Increased recipient mortality risk \\
\hline Smoking & Increased recipient mortality risk \\
\hline
\end{tabular}

DCD, determination of death; DBD, donation after brain death; COPD, chronic obstructive pulmonary disease; $\alpha$ ATD, alpha1-antitrypsin deficiency; ILD, interstitial lung disease; HLA, Human leukocyte antigen.

\section{Acknowledgments}

Funding: None.

\section{Footnote}

Provenance and Peer Review: This article was commissioned by the Guest Editor (George Makdisi) for the series "Lung Transplant: Current Status and Challenges" published in Current Challenges in Thoracic Surgery. The article has undergone external peer review.

Reporting Checklist: The author has completed the Narrative Review reporting checklist. Available at https://ccts. amegroups.com/article/view/10.21037/ccts-21-2/rc

Conflicts of Interest: The author has completed the
ICMJE uniform disclosure form (available at https://ccts. amegroups.com/article/view/10.21037/ccts-21-2/coif). The series "Lung Transplant: Current Status and Challenges" was commissioned by the editorial office without any funding or sponsorship. The author has no other conflicts of interest to declare.

Ethical Statement: The author is accountable for all aspects of the work in ensuring that questions related to the accuracy or integrity of any part of the work are appropriately investigated and resolved.

Open Access Statement: This is an Open Access article distributed in accordance with the Creative Commons Attribution-NonCommercial-NoDerivs 4.0 International License (CC BY-NC-ND 4.0), which permits the non- 
commercial replication and distribution of the article with the strict proviso that no changes or edits are made and the original work is properly cited (including links to both the formal publication through the relevant DOI and the license). See: https://creativecommons.org/licenses/by-nc-nd/4.0/.

\section{References}

1. Valapour M, Lehr CJ, Skeans MA, et al. OPTN/SRTR 2018 Annual Data Report: Lung. Am J Transplant 2020;20 Suppl s1:427-508.

2. Chaney J, Suzuki Y, Cantu E 3rd, et al. Lung donor selection criteria. J Thorac Dis 2014;6:1032-8.

3. Botha P, Rostron AJ, Fisher AJ, et al. Current strategies in donor selection and management. Semin Thorac Cardiovasc Surg 2008;20:143-51.

4. Aigner C, Winkler G, Jaksch P, et al. Extended donor criteria for lung transplantation--a clinical reality. Eur J Cardiothorac Surg 2005;27:757-61.

5. Fischer S, Gohrbandt B, Struckmeier P, et al. Lung transplantation with lungs from donors fifty years of age and older. J Thorac Cardiovasc Surg 2005;129:919-25.

6. Israni AK, Zaun D, Hadley N, et al. OPTN/SRTR 2018 Annual Data Report: Deceased Organ Donation. Am J Transplant 2020;20 Suppl s1:509-41.

7. Van Raemdonck D, Neyrinck A, Verleden GM, et al. Lung donor selection and management. Proc Am Thorac Soc 2009;6:28-38.

8. Cypel M, Yeung JC, Liu M, et al. Normothermic ex vivo lung perfusion in clinical lung transplantation. $\mathrm{N}$ Engl J Med 2011;364:1431-40.

9. Sharma A, Peltz M, Wait MA, et al. The conduct of thoracic organ procurement. Asian Cardiovasc Thorac Ann 2020;28:158-67.

10. Copeland H, Hayanga JWA, Neyrinck A, et al. Donor heart and lung procurement: A consensus statement. J Heart Lung Transplant 2020;39:501-17.

11. Reyes KG, Mason DP, Thuita L, et al. Guidelines for donor lung selection: time for revision? Ann Thorac Surg 2010;89:1756-64; discussion 1764-5.

12. Thabut G, Mal H, Cerrina J, et al. Influence of donor characteristics on outcome after lung transplantation: a multicenter study. J Heart Lung Transplant 2005;24:1347-53.

13. Chang SH, Kreisel D, Marklin GF, et al. Lung Focused Resuscitation at a Specialized Donor Care Facility Improves Lung Procurement Rates. Ann Thorac Surg 2018;105:1531-6.
14. Mascia L, Pasero D, Slutsky AS, et al. Effect of a lung protective strategy for organ donors on eligibility and availability of lungs for transplantation: a randomized controlled trial. JAMA 2010;304:2620-7.

15. Chambers DC, Zuckermann A, Cherikh WS, et al. The International Thoracic Organ Transplant Registry of the International Society for Heart and Lung Transplantation: 37 th adult lung transplantation report - 2020; focus on deceased donor characteristics. J Heart Lung Transplant 2020;39:1016-27.

16. Okamoto T, Omara M, Ahmad U, et al. Utilization of Marginal Lung Donors With Low $\mathrm{PaO} 2 / \mathrm{FiO} 2$ Ratio and High Body Mass Index. Ann Thorac Surg 2020;109:1663-9.

17. Benazzo A, Schwarz S, Frommlet F, et al. Donor ventilation parameters as predictors for length of mechanical ventilation after lung transplantation: Results of a prospective multicenter study. J Heart Lung Transplant 2021;40:33-41.

18. Orens JB, Boehler A, de Perrot M, et al. A review of lung transplant donor acceptability criteria. J Heart Lung Transplant 2003;22:1183-200.

19. Baldwin MR, Peterson ER, Easthausen I, et al. Donor age and early graft failure after lung transplantation: a cohort study. Am J Transplant 2013;13:2685-95.

20. Hall DJ, Jeng EI, Gregg JA, et al. The Impact of Donor and Recipient Age: Older Lung Transplant Recipients Do Not Require Younger Lungs. Ann Thorac Surg 2019;107:868-76.

21. Meyer DM, Bennett LE, Novick RJ, et al. Effect of donor age and ischemic time on intermediate survival and morbidity after lung transplantation. Chest 2000;118:1255-62.

22. Mulvihill MS, Gulack BC, Ganapathi AM, et al. The association of donor age and survival is independent of ischemic time following deceased donor lung transplantation. Clin Transplant 2017. doi: 10.1111/ ctr.12993.

23. Bittle GJ, Sanchez PG, Kon ZN, et al. The use of lung donors older than 55 years: a review of the United Network of Organ Sharing database. J Heart Lung Transplant 2013;32:760-8.

24. Choi AY, Jawitz OK, Raman V, et al. Predictors of Older Donor Lung Use: Are We Too Good at Saying No? Ann Thorac Surg 2020;110:1683-90.

25. Van Raemdonck D, Keshavjee S, Levvey B, et al. Donation after circulatory death in lung transplantation-five-year follow-up from ISHLT Registry. J Heart Lung Transplant 
2019;38:1235-45.

26. Chambers DC, Cherikh WS, Harhay MO, et al. The International Thoracic Organ Transplant Registry of the International Society for Heart and Lung Transplantation: Thirty-sixth adult lung and heart-lung transplantation Report-2019; Focus theme: Donor and recipient size match. J Heart Lung Transplant 2019;38:1042-55.

27. Bonde PN, Patel ND, Borja MC, et al. Impact of donor lung organisms on post-lung transplant pneumonia. J Heart Lung Transplant 2006;25:99-105.

28. Avlonitis VS, Krause A, Luzzi L, et al. Bacterial colonization of the donor lower airways is a predictor of poor outcome in lung transplantation. Eur J Cardiothorac Surg 2003;24:601-7.

29. Bunsow E, Los-Arcos I, Martin-Gómez MT, et al. Donorderived bacterial infections in lung transplant recipients in the era of multidrug resistance. J Infect 2020;80:190-6.

30. Inci I. Donors after cardiocirculatory death and lung transplantation. J Thorac Dis 2017;9:2660-9.

31. Levvey B, Keshavjee S, Cypel M, et al. Influence of lung donor agonal and warm ischemic times on early mortality: Analyses from the ISHLT DCD Lung Transplant Registry. J Heart Lung Transplant 2019;38:26-34.

32. Puri V, Scavuzzo M, Guthrie T, et al. Lung transplantation and donation after cardiac death: a single center experience. Ann Thorac Surg 2009;88:1609-14; discussion 1614-5.

33. Sabashnikov A, Patil NP, Popov AF, et al. Long-term results after lung transplantation using organs from circulatory death donors: a propensity score-matched analysis $†$. Eur J Cardiothorac Surg 2016;49:46-53.

34. Ruttens D, Martens A, Ordies S, et al. Short- and Longterm Outcomes After Lung Transplantation From Circulatory-Dead Donors: A Single-Center Experience. Transplantation 2017;101:2691-4.

35. Snell GI, Levvey BJ, Henriksen A, et al. Donor Lung Referrals for Lung Transplantation: A 'Behind The Scenes' View. Heart Lung Circ 2020;29:793-9.

36. Whitson BA, Hertz MI, Kelly RF, et al. Use of the donor lung after asphyxiation or drowning: effect on lung transplant recipients. Ann Thorac Surg 2014;98:1145-51.

37. Ciccone AM, Stewart KC, Meyers BF, et al.

Does donor cause of death affect the outcome of lung transplantation? J Thorac Cardiovasc Surg 2002;123:429-34; discussion 434-6.

38. Singhal AK, Sheng X, Drakos SG, et al. Impact of donor cause of death on transplant outcomes: UNOS registry analysis. Transplant Proc 2009;41:3539-44.
39. Oto T, Griffiths A, Levvey B, et al. Donor history of asthma is not a contraindication to lung transplantation: 12-year single-center experience. J Heart Lung Transplant 2004;23:309-16.

40. De Wolf J, Renard R, Lehouerou T, et al. Hanging donor lungs give good short-, mid- and long-term results in lung transplantation. Clin Transplant 2020;34:e13758.

41. Ananiadou O, Schmack B, Zych B, et al. Suicidal hanging donors for lung transplantation: Is this chapter still closed? Midterm experience from a single center in United Kingdom. Medicine (Baltimore) 2018;97:e0064.

42. Peltz M, Edwards LB, Jessen ME, et al. HLA mismatches influence lung transplant recipient survival, bronchiolitis obliterans and rejection: implications for donor lung allocation. J Heart Lung Transplant 2011;30:426-34.

43. Conte JV Jr, Ferber LR, Borja M, et al. Lung transplantation from dialysis dependent donors. J Heart Lung Transplant 2000;19:894-6.

44. Bush A, Gabriel R. Pulmonary function in chronic renal failure: effects of dialysis and transplantation. Thorax 1991;46:424-8.

45. Palleschi A, Mendogni P, Tosi D, et al. Lung Transplantation From Donors After Previous Cardiac Surgery: Ideal Graft in Marginal Donor? Transplant Proc 2017;49:686-91.

46. Brugière $\mathrm{O}$, Thabut $\mathrm{G}$, Suberbielle $\mathrm{C}$, et al. Relative impact of human leukocyte antigen mismatching and graft ischemic time after lung transplantation. J Heart Lung Transplant 2008;27:628-34.

47. Konheim JA, Kon ZN, Pasrija C, et al. Predictive equations for lung volumes from computed tomography for size matching in pulmonary transplantation. J Thorac Cardiovasc Surg 2016;151:1163-9.e1.

48. Park S, Ring W, Wait MA. Lung Volumes Pre and Post Transplant: The Frustum Model. J Heart Lung Transplant 2017;36:S158.

49. Eberlein M, Reed RM, Maidaa M, et al. Donor-recipient size matching and survival after lung transplantation. A cohort study. Ann Am Thorac Soc 2013;10:418-25.

50. Mason DP, Batizy LH, Wu J, et al. Matching donor to recipient in lung transplantation: How much does size matter? J Thorac Cardiovasc Surg 2009;137:1234-40.e1.

51. Barnard JB, Davies O, Curry P, et al. Size matching in lung transplantation: an evidence-based review. J Heart Lung Transplant 2013;32:849-60.

52. Garfein ES, McGregor CC, Galantowicz ME, et al. Deleterious effects of telescoped bronchial anastomosis in single and bilateral lung transplantation. Ann Transplant 
2000;5:5-11.

53. Raja S, Murthy SC, Pettersson GB, et al. Managing extreme airway size mismatch in lung transplantation: the "upper lobectomy" technique. Semin Thorac Cardiovasc Surg 2011;23:336-8.

54. Bonser RS, Taylor R, Collett D, et al. Effect of donor smoking on survival after lung transplantation: a cohort study of a prospective registry. Lancet 2012;380:747-55.

55. Okahara S, Levvey B, McDonald M, et al. Influence of the donor history of tobacco and marijuana smoking on early and intermediate lung transplant outcomes. J Heart Lung Transplant 2020;39:962-9.

56. MacGowan GA, Dark JH, Corris PA, et al. Effects of drug abuse, smoking and alcohol on donor hearts and lungs. Transpl Int 2019;32:1019-27.

57. Aslam S, Grossi P, Schlendorf KH, et al. Utilization of hepatitis $\mathrm{C}$ virus-infected organ donors in cardiothoracic transplantation: An ISHLT expert consensus statement. J Heart Lung Transplant 2020;39:418-32.

58. Woolley AE, Piechura LM, Goldberg HJ, et al. The impact of hepatitis $\mathrm{C}$ viremic donor lung allograft characteristics on post-transplantation outcomes. Ann Cardiothorac Surg 2020;9:42-8.

59. Yusen RD, Edwards LB, Kucheryavaya AY, et al. The registry of the International Society for Heart and Lung Transplantation: thirty-first adult lung and heart-lung transplant report--2014; focus theme: retransplantation. J Heart Lung Transplant 2014;33:1009-24.

doi: $10.21037 /$ ccts-21-2

Cite this article as: Peltz M. A narrative review of the impact of donor factors and selection criteria on outcomes after lung transplantation. Curr Chall Thorac Surg 2021.
60. Halloran K, Aversa M, Tinckam K, et al. Comprehensive outcomes after lung retransplantation: A single-center review. Clin Transplant 2018;32:e13281.

61. Ren D, Kaleekal TS, Graviss EA, et al. Retransplantation Outcomes at a Large Lung Transplantation Program. Transplant Direct 2018;4:e404.

62. Riou B, Guesde R, Jacquens Y, et al. Fiberoptic bronchoscopy in brain-dead organ donors. Am J Respir Crit Care Med 1994;150:558-60.

63. Botha P, Fisher AJ, Dark JH. Marginal lung donors: A diminishing margin of safety? Transplantation 2006;82:1273-9.

64. Ayyat KS, Okamoto T, Niikawa H, et al. DireCt Lung Ultrasound Evaluation (CLUE): A novel technique for monitoring extravascular lung water in donor lungs. J Heart Lung Transplant 2019;38:757-66.

65. Costa J, Sreekanth S, Kossar A, et al. Donor lung assessment using selective pulmonary vein gases. Eur J Cardiothorac Surg 2016;50:826-31.

66. Banga A, Mohanka M, Mullins J, et al. Hospital length of stay after lung transplantation: Independent predictors and association with early and late survival. J Heart Lung Transplant 2017;36:289-96.

67. Loor G, Radosevich DM, Kelly RF, et al. The University of Minnesota Donor Lung Quality Index: A ConsensusBased Scoring Application Improves Donor Lung Use. Ann Thorac Surg 2016;102:1156-65. 